Z. klin. Chem. u. klin. Biochem.

э. Jg., S. 486-488, November 1971

\title{
Anmerkungen zur Methodik der quantitativen gaschromatographischen Zụckeranalyse
}

\author{
Von B. SALFNER \\ Aus der Abteilung Immunbiologie (Leiter: Prof. Dr. G. Ublenbruck) der Medizinischen \\ Universitätsklinik Köln-Lindenthal
}

(Eingegangen am 8. Juli 1971)

Die Arbeit beschreibt methodische Verbesserungen der quantitativen gaschromatographischen Zuckeranalyse. Die Ånderungen betreffen die Hydrolyse, die Gleichgewichtseinstellung der Zuckeranomeren und die N-Reacetylierung der Aminozucker. Die Methode wird mit Hilfe der Analyse von Standard-Zuckergemischen geprüft.

\section{Observations on the quantitative analysis of carbobydrates by gas-liquid chromatography}

The quantitative analysis of sugars by gas-liquid chromatography was improved by the modification of the hydrolysis, the equilibration of sugar anomers and the N-reacetylation of hexosamines. The method was tested with standard mixtures of carbohydrates.

In einer früheren Arbeit (1) haben wir die gaschromatographische Analyse des Kohlenhydratanteiles serologisch aktiver Glykoproteine beschrieben. Wir versuchten dabei darzustellen, daß die quantitative Analyse komplexer Kohlenhydratketten trotz bestehender Fehlermöglichkeiten mit ausreichender Genauigkeit durchzuführen ist, da wir geringe Verunreinigungen der zur Analyse verwendeten Ausgangssubstanzen nicht ausschließen konnten. Die vorliegende Arbeit baut auf den Erfahrungen der ersten Arbeit auf und gibt einen Überblick über die methodischen Verbesserungen, die für die exakte Analyse reiner Substanzen erforderlich sind. Die Methode wurde im wesentlichen in drei Punkten geändert:

1. in der Auswahl der hydrolytischen Bedingungen

2. in der Einführung eines Abschnittes für die Gleichgewichtseinstellung der Anomeren

3. in der N-Reacetylierung der Aminozucker.

\section{Auswahl der Hydrolysebedingungen}

Von verschiedenen Untersuchern wird bestätigt $(2,3)$, $\mathrm{da} B$ es die optimale Hydrolysebedingung, die für alle Monosaccharide eines Glykoproteins gleichermaßen geeignet ist, nicht gibt. $\mathrm{Da}$ die zur Verfügung stehende Substanzmenge meist gering ist, müssen wir uns in der Regel auf zwei parallel durchgeführte Analysen beschränken. Wir führen neben einer Hydrolyse mit wasserfreier methanolischer $\mathrm{HCl}(1,5-2 \mathrm{~N} \mathrm{HCl}$ für $3 \mathrm{Stdn}$. bei $100^{\circ}$ ) eine zweite Hydrolyse in wäßr. $\mathrm{HCl}$ bei höherer Säurekonzentration durch $(3-4 \mathrm{~N} \mathrm{HCl}$ für 3 Stdn. bei $100^{\circ}$ ). Methylglykoside erscheinen uns aus den beschriebenen Gründen (1) für die Analyse von Hexosen am geeignetsten. Die Hydrolyse mit wäßr. $\mathrm{HCl}$ ist für die Bestimmung von Aminozuckern notwendig, da erstens die Uberführung der Hexosamine in Methylglykoside unvollständig ist und zweitens die zur vollständigen glykosidischen Spaltung der Hexosamine erforderlichen hohen Säurekonzentrationen bei der Methanolyse zu Ringisomerisierungen und damit zu einer Vermehrung der Peaks führen $(1,3,4)$. Die von uns zur Hydrolyse eingesetzten Substanzmengen betragen 2-5 mg. Die methodische Durchführung der Methanolyse wurde bereits beschrieben, die Hydrolyse in wäßr. $\mathrm{HCl}$ unterscheidet sich methodisch nicht.

\section{Einstellung des Anomerengleichgewichtes}

Für die Überführung der Monosaccharide in flüchtige Derivate stehen mehrere Verfahren zur Auswahl. Am häufigsten verwandt werden jedoch TrimethylsilylDerivate und Alditolacetat-Derivate, vor allem wohl deshalb, weil sie am leichtesten quantitativ aufbereitet werden können. Trimethylsilyl-Derivate haben einige Vorteile gegenüber Polyalkoholen von Monosacchariden:

1. die Aufbereitung ist einfacher und zeitsparender

2. Trimethylsilyl-Derivate von Aldohexosen werden auf vergleichbaren Säulen besser getrennt

3. Trimethylsilyl-Derivate exlauben im Gegensatz zu Alditolacetat-Derivaten die Analyse von Ketozuckern.

Das Problem bei der Analyse von TrimethylsilylZuckern ist jedoch die Anomerentrennung. Eine vollständige Trennung sämtlicher Anomeren ist bisher nicht gelungen. Bei unseren Analysen treten Uberlagerungen der Peaks von $\beta$-Fucose/ $\alpha$-Xylose, $\beta$ Galaktose/ $\alpha$-Glucose und $\beta$-N-Acetyl-Galaktosamin $/ \beta$ N-Acetyl-Glucosamin auf. Die quantitative Analyse dieser Zucker ist nur unter Einbeziehung eines konstanten Anomerengleichgewichts in die Berechnung möglich. Die Zeitdauer der Gleichgewichtseinstellung der in $\mathrm{H}_{2} \mathrm{O}$ gelösten Monosaccharide wird am Beispiel der Glucose aus Tabelle 1 ersichtlich. Das Verhalten der übrigen Monosaccharide ist dem der Glucose ver- 
Tab. 1

Einstellung des Mutarotationsgleichgewichts Anomerenverhältnis der Glucose nach verschiedenen Zeiten der Aquilibrierung in dest. Wasser

\begin{tabular}{ccc}
$\begin{array}{c}\text { Dauer } \\
\text { [Min.] }\end{array}$ & \multicolumn{2}{c}{$\begin{array}{c}\text { Glucose-Anomer } \\
{[\% \text { der Peakfläche] }}\end{array}$} \\
& $\alpha-$ & $\beta-$ \\
\hline 15 & 84,43 & 15,57 \\
30 & 54,74 & 45,26 \\
60 & 47,33 & 52,67 \\
90 & 46,15 & 53,85 \\
120 & 44,84 & 55,16 \\
180 & 41,98 & 58,02 \\
& 41,70 & 58,30 \\
\hline
\end{tabular}

gleichbar. Das Anomerengleichgewicht hat sich nach etwa 2 Stunden eingestellt. Aus diesen Untersuchungen leiten wir die Forderung nach der Zwischenschaltung einer 2-3stündigen Einstellungszeit im Anschluß an die Hydrolyse ab. Es ist nämlich durchaus vorstellbar, daß Monosaccharide, die erst gegen Ende der Hydrolyse glykosidisch gespalten werden, bei „zügiger" Aufbereitung der Substanz die Einstellung des Gleichgewichts nicht erreichen. Die Durchführung der Probenvorbereitung und der Zeitpunkt der Zwischenschaltung der Einstellungszeit für das Anomerengleichgewicht ist aus Schema $1 \mathrm{zu}$ ersehen.

Schema 1

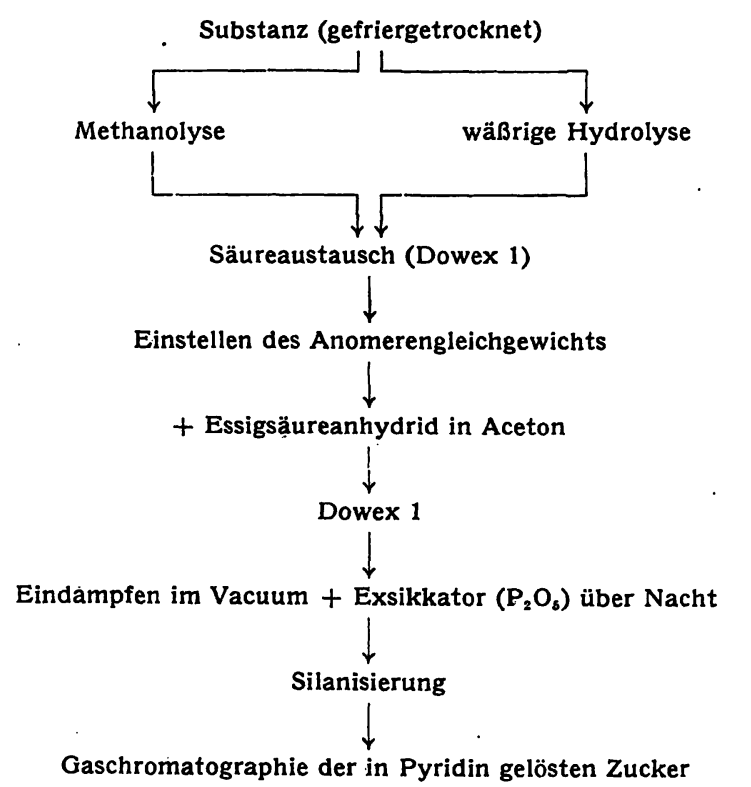

\section{N-Reacetylierung der Aminozucker}

Die von uns bisher angewandten Methoden der NReacetylierung $(1,5,6)$ sind in ihrem Ergebnis unbefriedigend, die Acetylierungsquote liegt dabei unter $50 \%$. LEVVY und Mitarbeiter (3) beschrieben nun eine neue Methode, die eine nahezu vollständige N-Acetylierung gewährleisten soll. Die Vollständigkeit dieser Reaktion ist für die exakte quantitative Analyse einer Substanz aus zwei Gründen unumgänglich. Einmal ist die Silanisierung nicht acetylierter Aminozucker unvollständig $(60 \% !)$, zum anderen ist eine quantitative Berechnung durch Überlagerungen der Peaks nicht möglich.

\section{Arbeitsvorsclbrift}

Etwa $2 \mathrm{mg}$ Hexosamin- $\mathrm{HCl}$ bzw. ein Hexosamin-HexosenGemisch werden in $1,75 \mathrm{ml} 3 \mathrm{~N} \mathrm{HCl}$ gelöst. Danach wird in einem zugeschmolzenen, dickwandigen Röhrchen unter $\mathrm{N}_{2}$ für $3 \mathrm{Stdn}$. bei $100^{\circ}$ hydrolysiert. Uberführen in ein Reagenzglas und Zugabe von $3,25 \mathrm{ml}$ dest. Wasser. In einem Parallelversuch werden unter Umgehung der Hydrolyse 3,25 $\mathrm{ml}$ dest. Wasser sofort zu der in $\mathrm{HCl}$ gelösten Substanz gegeben.

Die Beseitigung der Säure erfolgt mit Hilfe des Anionenaustauschers Dowex $1 \times 4$ (Carbonat-Form; Säule $1 \times 6 \mathrm{~cm}$ ). Die Substanz wird in kleinen Portionen aufgetragen. Wiederholtes Beklopfen det Säule ist notwendig, damit das entstehende $\mathrm{CO}_{2}$ entweichen kann. Dowex 1 wurde mit $1 \mathrm{M} \mathrm{Na}_{2} \mathrm{CO}_{3}$ in die Carbonatform überführt. Die Eluierung erfolgt mit dest. Wasser, der $\mathrm{pH}-$ Wert des Eluates wird wiederholt gemessen. Die ersten $2 \mathrm{ml}$ werden verworfen, die nächsten $16 \mathrm{ml}$ gesammelt. Die gesammelte Fraktion wird auf $5 \mathrm{ml}$ cingeengt. Nach Einstellen des Gleichgewichtes der Anomeren (2-3 Stdn.) wird mit dem gleichen Volumen 0,6proz. (v/v) Essigsäureanhydrid in Aceton versetzt. Das Gemisch wird sorgfältig geschüttelt, 1/2 Std. bei Raumtemperatur stehen gelassen und dann auf eine zweite Säule des gleichen Austauschers aufgetragen. Die zweite Säule wurde zuvor mit 50proz. Aceton in Wasser gespült, die Eluierung erfolgt mit dest. Wasser. Die ersten $5 \mathrm{ml}$ werden verworfen, die folgenden $32 \mathrm{ml}$ gesammelt. Nach Zugabe des gelösten Standards (Erythrit 0,5-1 mg) wird die gesammelte Fraktion am Rotationsverdampfer zur Trockne eingeengt. Entfernung von Feuchtigkeitsresten im Exsikkator übcr $\mathrm{P}_{2} \mathrm{O}_{5}$ für $12 \mathrm{Stdn}$.

\section{Gascbromatograpbie}

Die Silanisierung der Zucker erfolgt mit $1 \mathrm{ml}$ eines Gemisches von Pyridin, Hexamethyldisilazan (Fluka AG) und Trimethylchlorsilan (Serva, Heidelberg) im Verhältnis 7:3:2 (v/v). Das Gemisch wird wiederholt kräftig geschüttelt und $30 \mathrm{Min}$. bei Raumtemperatur stehen gelassen. Nach Abzentrifugieren der unlöslichen Anteile werden 1-2 $\mu \mathrm{l}$ der in Pyridin gelösten Zucker in die Säule eingespritzt.

Zur Analyse verwendeten wir einen Gaschromatographen der Firma Hewlett-Packard mit Glassäulen $(0,4 \mathrm{~cm}$ innerer $\varnothing, 240 \mathrm{~cm}$ Länge), Flammenionisationsdetektor und Temperaturprogrammierung. Für die Eluierung der Hexosamine ist eine Temperaturprogrammierung von $155^{\circ}$ bis $195^{\circ}$ erforderlich. Die quantitative Berechnung erfolgte durch Triangulierung der Peaks.

\section{Ergebnisse der $N$-Reacetylierung}

Die Verwendung eines Ionenaustauschers für die Eliminierung der $\mathrm{HCl}$ ist einer Neutralisation mit $\mathrm{Na}_{2} \mathrm{CO}_{3}$ oder $\mathrm{Ag}_{2} \mathrm{CO}_{3}$ vorzuziehen, da der Substanzverlust geringer ist. Die gaschromatographische Kontrolle ergibt eine vollständige Eluierung der Substanz aus der Säule. Die Sammelmenge von $16 \mathrm{ml}$ war bei dem verwendeten Säulenvolumen notwendig. Schwierigkeiten hatten wir bei hohen Säurekonzentrationen (über $3 \mathrm{~N}$ ) mit der Entfernung der $\mathrm{CO}_{2}$ aus den unteren Säulenabschnitten.

Die Methode von Levvy und Mitarbeitern hat zwei Besonderheiten gegenüber den uns bisher bekannten N-Reacetylierungsmethoden: die Lösung des Essigsäureanhydrids in Aceton und die Verwendung eines Anionenaustauschers. Untersuchungen der Teilschritte der Reaktion ergaben, daß die Reacetylierung der in Wasser gelösten, neutralen Substanz nach Zugabe des Essigsäureanhydrid-Aceton-Gemisches stattfinden kann. Der Grad der N-Acetylierung ist dabei jedoch reduziert und die quantitative Rückgewinnung gestört.

Tabelle 2 zeigt den Grad der N-Acetylierung freier Hexosamine. Aufgrund der Flächenberechnung der 
Peaks würde sich eine Acetylierung von etwa $97 \%$ ergeben. Bezieht man allerdings die unvollständige Silanisierung nicht acetylierter Aminozucker in die Berechnung ein, ergibt sich ein Prozentsatz von 91.

Tab. 2

$\mathrm{N}$-Acetylierung von Hexosaminen. Angabe in \% Peakfläche (nicht korrigiert) und unter Berücksichtigung der unvollständigen Silanisierung freier Hexosamine (korrigiert)

\begin{tabular}{|c|c|c|c|}
\hline & \multirow[b]{2}{*}{ Einwaage } & \multicolumn{2}{|c|}{$\begin{array}{c}\text { Hexosamin acetyliert } \\
\text { [\% der Peakfläche] }\end{array}$} \\
\hline & & $\underset{\text { korrigiert }}{\text { nicht }}$ & korrigiert \\
\hline $\begin{array}{l}\text { Glucosamin-HCl } \\
\text { Glucosamin-HCl } \\
\text { Galaktosamin-HCl } \\
\text { Galaktosamin-HCl }\end{array}$ & $\begin{array}{l}2,5 \mathrm{mg} \\
1,9 \mathrm{mg} \\
2,5 \mathrm{mg} \\
2,0 \mathrm{mg}\end{array}$ & $\begin{array}{l}96,76 \\
97,02 \\
97,0 \\
96,64\end{array}$ & $\begin{array}{l}90,68 \\
91,16 \\
91,11 \\
90,98\end{array}$ \\
\hline Durchschnittswert & & 98,86 & 90,98 \\
\hline
\end{tabular}

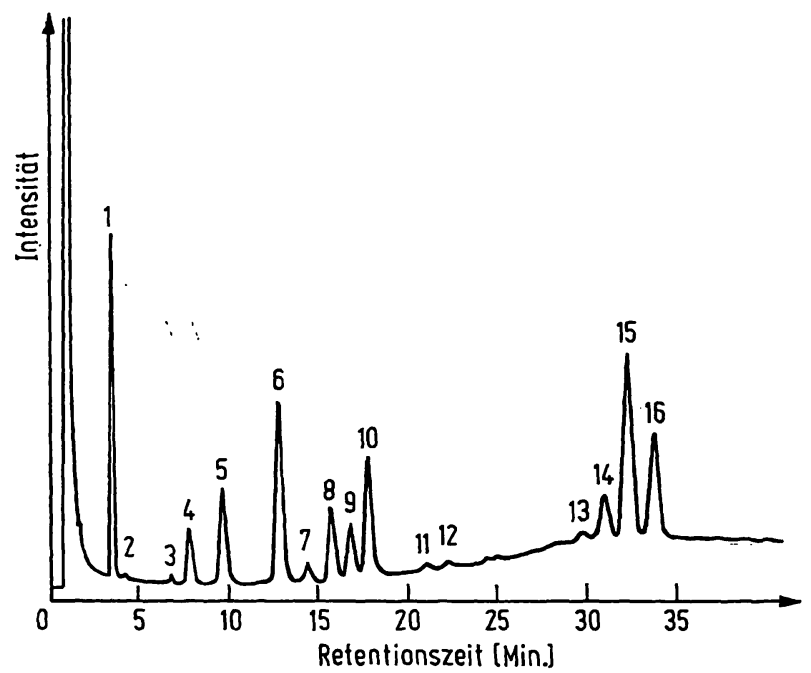

Abb. 1

Gaschromatogramm eines Standard-Zuckergemisches. Bedingungen: Säulenfüllung OV $17,3^{\circ} \% 155-195^{\circ}$, Programmierungsrate $4^{\circ} / \mathrm{Min}$. ab Eluierung der Mannose. Empfindlichkeit: $10^{3} / 8.1,2=$ Erythrit (interner Standard); 3-5 = Fucose; 6,9= Mannose; $7,8,10=$ Galaktose; $11=$ Galaktosamin; $12=$ Glucosamin; $13=\gamma$-NAC Galaktosamin; $14=\alpha-N A c-G a l a k t o s a m i n ; 15=\alpha-N A c-G l u c o s a m i n$ $16=\beta$-NAc-Galaktosamin $+\beta$-NAc-Glucosamin
Die Methode wurde weiterhin auf die Möglichkeit der quantitativen Rückgewinnung von Standard-Zuckergemischen geprüft. Die Ergebnisse sind in Tabelle 3 zusammengestellt. Die Genauigkeit der Analyse nimmt mit der Wiederholung der Versuche deutlich zu. Wir sind daher sicher, daß die quantitative Rückgewinnung der Zucker nach der N-Reacetylierung gewährleistet ist. Abbildung 1 zeigt das Chromatogramm eines Standard-Zuckergemisches.

\section{Diskussion}

Die Genauigkeit der quantitativen Analyse komplexer Glykoproteine wurde mit Hilfe der beschriebenen drei methodischen Verbesserungen so weit gesteigert, daß eine Analyse reiner Sübstanzen bei besčhränkten Substanzmengen mit einer Fehlerbreite unter $4 \%$ möglich ist. Die Reproduzierbarkeit der Ergebnisse ist am besten bei Durchführung beider Hydrolysearten. Bei Substanzmengen unter $4 \mathrm{mg}$ beschränken wir uns auf die Hydrolyse mit wäßriger $\mathrm{HCl}$, da die exakte Analyse der Hexosamine für unsere Fragestellungen in der Regel wichtiger ist. Wir nehmen dabei mögliche Verluste an Desoxyhexosen und Pentosen in Kauf. Der für eine Analyse benötigte Arbeits- und Zeitaufwand ist, besonders durch die N-Reacetylierung, größer geworden. $\mathrm{Da}$ die hierbei auftretenden methodischen Schwierigkeiten jedoch gering sind, halten wir eine Analysendauer von zwei Tagen für durchaus vertretbar. Schema 1 faßt den Gang der Analyse nochmals kurz zusammen.

Die Untersuchungen wurden durch ein Stipendium der Deutschen Forschungsgemeinschaft gefördert.

Herrn Prof. Dr. G. Uhlenbruck möchte ich für viele Anregungen und seinen Rat bei der Durchführung der Arbeit herzlich danken. Die Arbeit wurde im Rahmen des Sonderforschungsbereiches SFB 68/IV der Deutschen Forschungsgemeinschaft durchgeführt. Frau H. MEYER danke ich für geschickte technische Assistenz.

Tab. 3

N-Acetylierung von Hexosaminen und quantitative Wiederfindung der Komponenten dreier Monosaccharidgemische mit der Methode nach LEVVY

\begin{tabular}{|c|c|c|c|c|c|c|c|c|c|}
\hline Zucker & $\begin{array}{c}\text { eingesetzt } \\
\text { (mg) }\end{array}$ & $\begin{array}{c}\text { Gemisch I } \\
\text { gefunden } \\
\text { (mg) }\end{array}$ & $\begin{array}{c}\text { Fehler } \\
(\%)\end{array}$ & $\begin{array}{l}\text { eingesetzt } \\
\text { (mg) }\end{array}$ & $\begin{array}{l}\text { Gemisch II } \\
\text { gefunden } \\
\text { (mg) }\end{array}$ & $\begin{array}{c}\text { Fehler } \\
(\%)\end{array}$ & $\begin{array}{c}\text { eingesetzt } \\
\text { (mg) }\end{array}$ & $\begin{array}{c}\text { Gemisch III I } \\
\text { gefunden } \\
\text { (mg) }\end{array}$ & $\begin{array}{c}\text { Fehler } \\
(\%)\end{array}$ \\
\hline \multirow{2}{*}{$\begin{array}{l}\text { L-Fucose } \\
\text { D-Mannose } \\
\text { D-Galaktose } \\
\text { D-Glucose } \\
\text { NAc-Galaktosamin } \\
\text { NAc-Glucosamin }\end{array}$} & \multirow{2}{*}{$\begin{array}{l}0,9 \\
1,5 \\
1.8 \\
2,0 \\
1,2 \\
3,9\end{array}$} & \multirow{2}{*}{$\begin{array}{l}1,01 \\
1,502 \\
1,788 \\
1,92 \\
1,16 \\
3,75\end{array}$} & \multirow{2}{*}{$\begin{array}{l}+12,2 \\
+0,13 \\
-0,65 \\
-4,0 \\
-3,4 \\
-3,8\end{array}$} & $\begin{array}{l}1,0 \\
1,9\end{array}$ & $\begin{array}{l}1,087 \\
1,897\end{array}$ & $\begin{array}{l}+8,7 \\
-0,15\end{array}$ & 2,6 & 2,64 & $+1,54$ \\
\hline & & & & $\begin{array}{l}3,0 \\
1,7 \\
2,1\end{array}$ & $\begin{array}{l}2,863 \\
1,62 \\
2,02\end{array}$ & $\begin{array}{l}-4,57 \\
-4,7 \\
-3,8\end{array}$ & 1,5 & 1,458 & $-2,8$ \\
\hline
\end{tabular}

\section{Literatur}

1. SalfNer, B. und G. UhLenbruck, diese Z. 9, 95 (1971). -

2. Graham, E. R. B. und A. Neuberger, Biochem. J. 106, 593 (1968). - 3. Levvy, G. A., A. J. Hax, J. Conchie und I. StraCHAN, Biochim. biophysica Acta, Amsterdam 222, 333 (1970). 4. Radhakrishnamurthy, B., E. R. Dalferes, G. S. Berenson,
Analytic. Biochem. 17, 545 (1966). - 5. Sweeley, C. C., R Bentrex, M. Makita und W. W. Wells, J. Amer. chem. Soc. 85, 2497 (1963). - 6. Clamp, J. R., G. Dawson und L. Hovgh, Biochim. biophysica Acta, Amsterdam 148, 342 (1967).

Dr. B. Salfner

Abteilung Immunbiologie det Medizinischen Klinik $5 \mathrm{Köln} 41$

Kerpenerstr. 15 
Der zur Förderung der Forschung gestiftete

\section{HEINRICH - WIELAND - PREIS}

wird hiermit satzungsgemäß für das Jahr 1972 ausgeschrieben.

Der Preis, benannt nach dem 1957 verstorbenen Nobelpreisträger Professor Dr. Heinrich Wieland, ist für Arbeiten aus der Chemie, Biochemie und Physiologie der Fette und Lipoide sowie über deren ernährungsphysiologische und klinische Bedeutung ausgesetzt und wird jährlich verliehen.

Der HEINRICH-WIELAND-PREIS besteht aus einer "Heinrich-WielandPlakette« und einem Geldbetrag in Höhe von 10000 DM.

Ein Kuratorium, dem zur Zeit die Herren

Prof. Dr. Werner Droese, Dortmund Prof. Dr. Rudolf Pannhorst, Berlin

Prof. Dr. Werner Heimann, Karlsruhe Prof. Dr. Gotthard Schettler, Heidelberg

Prof. Dr.Joachim Kühnau, Hamburg Prof. Dr. Theodor Wieland, Heidelberg

Prof. Dr. Dr.K. Lang, Bad Krozingen Prof. Dr. Viktor Wolf, Hamburg

Prof. Dr. Nepomuk Zöllner, München

angehören, wird den Preisträger auswählen.

Einsendeberechtigt für die Verleihung des HEINRICH-WIELAND-PREISES für das Jahr 1972 sind Autoren von unveröffentlichten oder in den Jahren 1970 bis 1972 publizierten wissenschaftlichen Arbeiten. Der eingereichte Beitrag muß in deutscher, englischer oder französischer Sprache abgefaßt sein. Bei fremdsprachlichen Arbeiten ist eine Zusammenfassung (etwa 3 Seiten) in deutscher Sprache erforderlich. Abhandlungen, die bereits mit einem anderen wissenschaftlichen Preis ausgezeichnet sind, können nicht prämiiert werden.

Einsendeschluß für die Verleihung im Jahre 1972 ist der 1. März 1972.

Die Arbeiten sind in einem Exemplar bis zu diesem Datum an folgende Anschrift einzusenden:

Kuratorium für die Verleihung des HEINRICH-WIELAND-PREISES Im Auftrag Prof. Dr. Alfons Fricker, 7501 Grötzingen, Ringelberghohl 12 


\section{PYROSZINT FHT 5030}

ein Verbrennungsautomat

zur Probenvorbereitung

für die Flüssigszintillationsmessung

In einem 1-I-Kolben können auf einem Papierträger von ca. $130 \mathrm{mg}$ Probenmengen bis zu $100 \mathrm{mg}$ verbrannt werden. Verwendet man leichtere Papierträger (Seidenpapier), kann die Probenmenge auf $150-200 \mathrm{mg}$ gesteigert werden.

An das Probenmaterial wird lediglich die Anforderung der Brennbarkeit gestellt, es ist gleichgültig, ob das Material ein starker chemischer oder Farb-Löscher ist, oder ob es sich um in Szintillator unlösliches Material - z. B. biologischer Herkunft- handelt, Auch Flüssigproben, deren Radioaktivität nicht flüchtig ist, können nach Trocknen mit diesem Verfahren bearbeitet werden. Auch ist bei Verwendung eines speziellen Szintillator-Absorbensgemisches die Radioaktivitätsanalyse von 3H/14C-markiertem Material direkt an einer Probe möglich.

Da für einen Zyklus am Pyroszint ca. 25 min. benötigt werden, können somit inklusive Absorptionszeif 8 Proben in ca. $35 \mathrm{~min}$. verarbeitet werden.
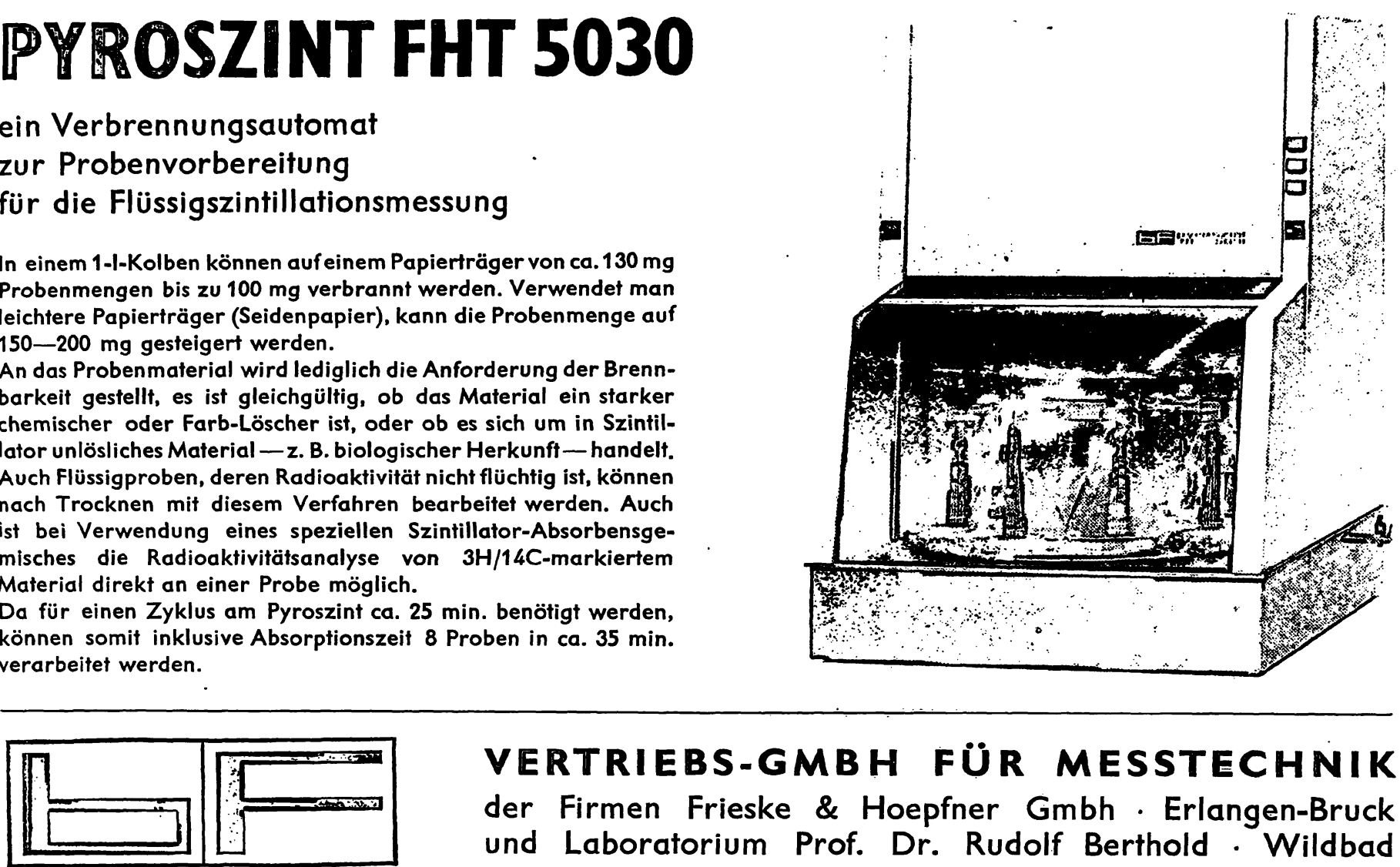

VERTRIEBS-GMBH FÜR MESSTECHNIK der Firmen Frieske \& Hoepfner Gmbh . Erlangen-Bruck und Laboratorium Prof. Dr. Rudolf Berthold - Wildbad

75 Karlsruhe 41 - Bergwaldstraße 30 . Postfach 76 - Tel. (0721) $401011-14 \cdot$ Telex $7825927 \cdot$ Telegramm Raytec

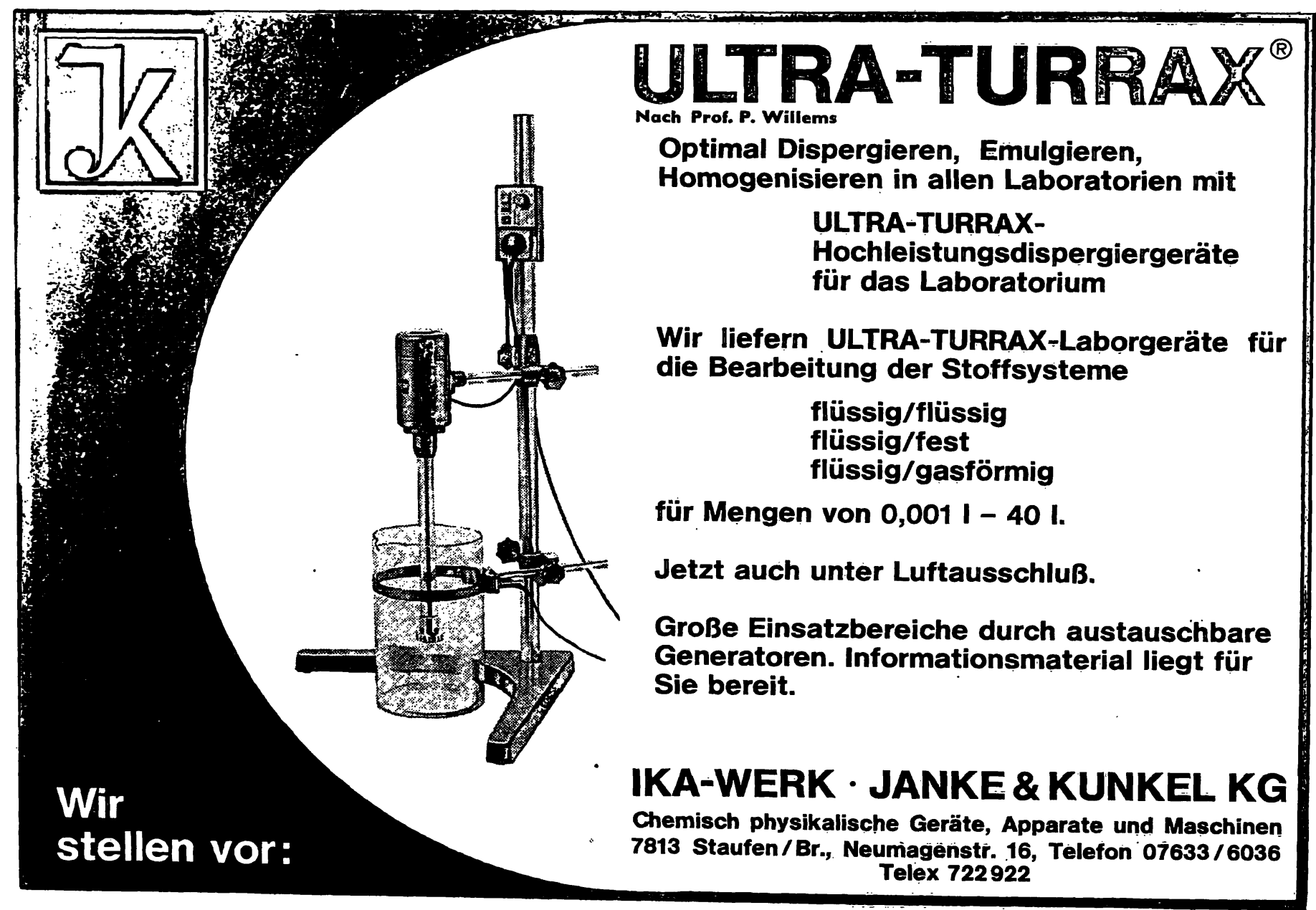

